\title{
Study on Measurement of Spatial Motion Trajectory Based on Laser Tracing Measurement System
}

\author{
Dazhong Guo ${ }^{1, a}$, Yuanyuan Zou ${ }^{2, b}$, Sijun Zhu ${ }^{3, c}$, Mingyang Zhao ${ }^{4, d}$ \\ 1,2,3,4 Shenyang Institute of Automation, Chinese Academy of Sciences Shenyang 110016, China \\ adzguo@sia.cn, byyzou@sia.cn, c'sjzhu@sia.cn, ${ }^{\mathrm{d}} \mathrm{myzhao@sia.cn}$
}

\begin{abstract}
Keywords: non-contact Measurement, laser tracing measurement system, Measurement of Spatial Motion Trajectory, laser interferometer
\end{abstract}

\begin{abstract}
In this paper an approach of measuring the positional precision of the execution terminal of the industrial robot is introduced, and the approach based on a non-contact laser tracing measurement has the advantage of high-speed response, high detection accuracy and non-contact measurement. This method utilizes the linear motion units and photo detection units to trace the target actively. The measurement of the target coordinates are measured with the laser interferometer linear measurement module and the grating ruler. This system can achieve the position measurement of the planar motion objects without contact in real-time condition and also possesses the high precision.
\end{abstract}

\section{Introduction}

With the fast development of both robotic technology, industrial robots widely employed in assembly, seam welding, testing, drilling and other fields, requirements of robots' positioning accuracy and kinematics performance have greatly improved. Recently, the position of robot end-effector is obtained through the position information of each joint. There are several serious problems. First, the robot's frame stiffness must be large, because any deflection of arms will lead to misjudge the position of the robot end-effector. The fact will increase the price and weight of machines; meanwhile limit the payload of robot. Second, even with the precision encoder and the solid component, the precision of large robot is low. The robot, which has a working space of $2 \mathrm{~m}$ and can transport different payload, usually has $1 \sim 2 \mathrm{~mm}$ of the absolute accuracy. To enable the robot to a high accuracy for those tasks, the measurement which can provide precise location information of robot has been required [1-4].

With wide measurement range, flexible, dynamic and high precision, the laser tracing measurement system can realize high-precision measurement of moving objects in space. Some companies, such as LEICA, API and FARO, have launched commercial laser tracking measurement system based on the spherical coordinate. National Metrology Institute of Japan (NRLM) and the British National Physical Laboratory (NPL) are developing measurement system based on the principle of multilateral laser tracking measurement system [5]; Tianjin University in China has developed laser tracking coordinate mapping system at first based on the multilateral method [6]. In relevant available products, gantry-type coordinate measuring machine has high accuracy, but the move should not be bulky; articulated arm measuring instrument is portable, but its error at $1 \mathrm{~m}$ is more than $30 \mu \mathrm{m}$, and generally it requires staff assistance, and it is not be independent Tracking; laser tracker is portable, but its error at $1 \mathrm{~m}$ is more than $15 \mu \mathrm{m}$, at the same time, its measurement accuracy is influenced by the accuracy of code disc and increased with the increase of the measuring distance.

In this paper, we study the laser tracing measurement technique based on Cartesian coordinates. This system overcomes the former's precision coupling. Then we use the system to test the position precision of the industry robot.

\section{Measuring principle and system structure}

To track the movement and determine its two-dimensional coordinates of a point in the plane, a target mirror is attached to the end-effector. The laser that the laser interferometer sent out reflects to the target mirror through the tracking mirror. Then the laser is divided into two beams to return to the light detection device and the interferometer by the beam splitter. 




Fig. 1. Measuring principle of laser tracking system

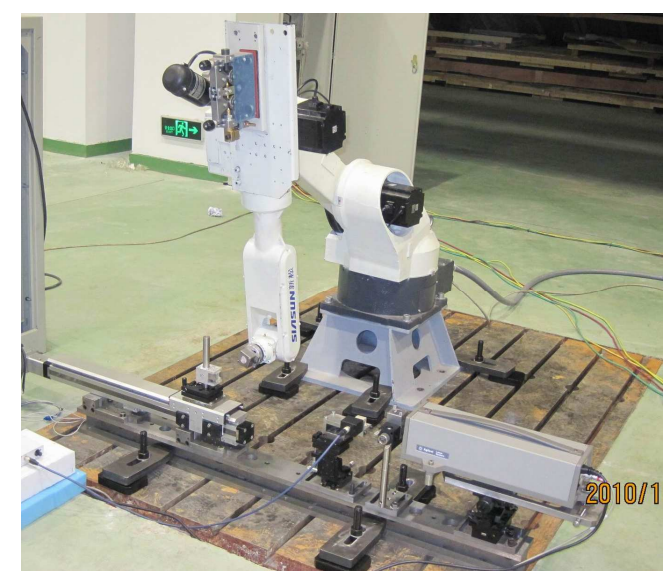

Fig. 2. Laser tracking measurement system

Though the interferometer monitors the changes of the fringes of two beams, the system can realize high-precision non-contact measurement. When the target moves, detection system measures the movement of the target mirror based on the position deviation of the return beam at the light detector. The tracking mirror adjusts the $\mathrm{X}$ position to re-aim the target, and we can measure the total displacement and the displacement of $\mathrm{X}$ direction through the laser interferometer and a grating ruler respectively in order to achieve the measurement of coordinates. The system consists of laser interferometer measurement system, tracking system, light detection system, control system and other subsystems. Its composition and spatial assembly relationship are shown in Figure 2.

\section{Measuring plan for position precision of robot}

The results at home and abroad showed that the flow in structural and moving parameters of the robot extends to the precision of end-effector. In this paper, load, position and velocity are the primary considerations. This paper takes welding robot as the example, analyses the position precision of the robot. The testing cube is selected in that portion of the working space with the greatest anticipated use. And the edges of the cube are parallel to the base coordinate system.
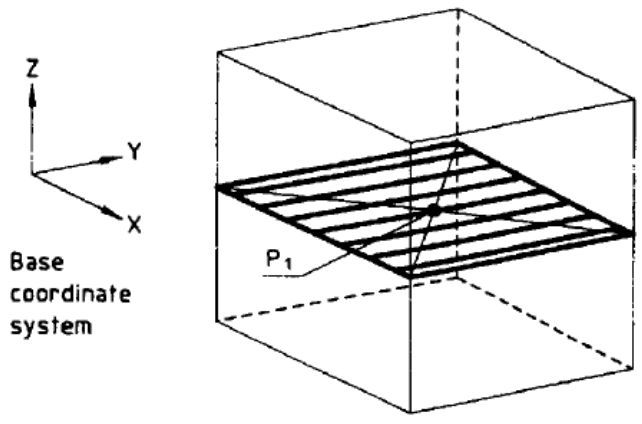

Fig. 3. Plan of location of test path

When employing robot to begin arc welding, it is usually carried out to teach the robot. Based on the inputted moving parameters, the arc welding robot executes the order again and again to meet the requirements of manufacturing line. Arc welding process requires the trajectory of end-effector to satisfy certain repeatability. When the track repeatability beyond technical requirements, the arc welding may lead to reduce the weld strength because of the deviation of weld center, and ultimately affect product quality. Thus, according to ISO9283 standard ${ }^{[7]}$, the major testing trajectories for the arc welding robot are lines and square. Spot welding process is different from arc welding process. Spot welding process requires the robot to keep position accuracy between the target points. So in the experimental measurement, it carried out only to measure the repeatability of the robot. When the position precision beyond the permitted range, it is also affect product quality due to weld defects. Therefore, the major testing trajectories for the arc welding robot and spot welding robot are points, lines and polylines. Selected cube is located in that portion of the working space with the greatest anticipated use, and its edges are parallel to the base coordinate system of robot. The test path is at the test plane of the cube, as is shown in Figure 3. Four measurement points are located on the diagonals of measuring plane. 
According to the requirements of ISO9283, test velocity can be chose $10 \%$ welding speed. And tests could be carried out at the maximum velocity achievable between the specified poses, i. e. with the velocity override set to $100 \%$, in each case. And additional test velocities are at $50 \%$ and/or $10 \%$ of this velocity. The research found that welding velocity is influenced by welding conveyor angle, plate thickness, and other factors, such as whether there is filler. In general, welding velocity is between $1.5 \mathrm{~m} / \mathrm{min} \sim 6 \mathrm{~m} / \mathrm{min}$. So test velocity should be between $0.15 \mathrm{~m} / \mathrm{min} \sim 0.6 \mathrm{~m} / \mathrm{min}$, that is $1.5 \mathrm{~m} / \mathrm{min} \sim$ $6 \mathrm{~m} / \mathrm{min}$. In this study, we select $5 \mathrm{~mm} / \mathrm{s}$ as the test velocities.

All tests are executed with no test load, and with a test load equal to $10 \%$ of rated load conditions. However, because the reflective component of test system are required to install at the body of end-effector, the experimental load for arc welding robot is actually a $1 \mathrm{~kg}$ and $6 \mathrm{~kg}$. The load for the spot welding robot is $1 \mathrm{~kg}$ and $16 \mathrm{~kg}$.

From the above, we can see position precision of robot is calculated by the test data from looping execution. Measurement system tracks the target mirror. At the same time, it snatches the feedback data from the functional units rapidly. Collection interval is approximately $15 \mathrm{~ms}$. Although such a data acquisition speed can guarantee the tracking performance and the request of real time, but result in large gathering samples. Take testing a square track of side length $200 \mathrm{~mm}$ as an example, test velocity is $5 \mathrm{~mm} / \mathrm{s}$, the number of loops is 30 , there needs to collect about 400k double-precision data, and file size is about $20 \mathrm{Mb}$. So huge amount of data Not only causes read difficultly, and conventional computer configuration cannot acquire the data quickly in execution unit of the system, even leads to memory leak. Considering inefficient analysis of a huge number of data, the system limit the number of loops not more than 10 times one group. In this test, the number of cycles is 3 .

The measure indices of positional precision are accuracy of position and repeatability of position. Accuracy of position expresses the deviation between a commend pose and the mean of the attained poses when approaching the command position from the same direction. The following factors are the main reasons: the accuracy of manufacturing mechanical parts of robot, the accuracy of assembling the constituting part of robot, the accuracy during the robot operation that is influenced by external forces, electronics system accuracy and motors operations, clearance existing in the system, wear behaviors, change in accuracy due to repair and change in the system accuracy during the preventive maintenance periodic programs. Repeatability of position expresses the closeness of agreement between the attained poses after $\mathrm{n}$ repeat visits to the same command position in the same direction. The measurement for repeatability requires:

- The same measurement procedure;

-The same observer;

-The same measuring instrument, used under the same conditions;

-The same location;

-Repetition over a short period of time.

The accuracy of position is as follows:

where

$$
A P_{P}=\sqrt{\left(\bar{x}-x_{c}\right)^{2}+\left(\bar{y}-y_{c}\right)^{2}+\left(\bar{z}-z_{c}\right)^{2}}
$$

$$
\bar{x}=\frac{1}{n} \sum_{j=1}^{n} x_{j}, \bar{y}=\frac{1}{n} \sum_{j=1}^{n} y_{j}, \bar{z}=\frac{1}{n} \sum_{j=1}^{n} z_{j}
$$

$\bar{x}, \bar{y}$ and $\bar{z}$ are the coordinates of the barycenter of the cluster of points obtained after repeating the same pose n times. $x_{c}, y_{c}$ and $z_{c}$ are the coordinates of the command pose. $x_{j}, y_{j}$ and $z_{j}$ are the coordinates of the $\mathrm{j}$-th attained pose.

Then repeatability of position is as follows:

$$
R P_{P}=\bar{l}+3 S_{l}
$$

where

$$
\bar{l}=\frac{1}{n} \sum_{j=1}^{n} l_{j} \quad l_{j}=\sqrt{\left(x_{j}-\bar{x}\right)^{2}+\left(y_{j}-\bar{y}\right)^{2}+\left(z_{j}-\bar{z}\right)^{2}} \quad S_{l}=\sqrt{\frac{\sum_{j=1}^{n}\left(l_{j}-\bar{l}\right)^{2}}{n-1}}
$$




\section{Measuring results}

In this stage, desired pose of end-effector is given to robot to go there. According to position coordinates of the end-effector in global reference frame, Mat lab is adopted to calculate the precision. As, the end-effector position in Z direction doesn't vary, the amount of error in $Z$ direction is considered to be zero. By comparing the ideal amounts of pose and real one, the positioning error are determined. The experimental results of accuracy of position (AP) and repeatability of position (RP) are shown in Table 1. Figure 4 shows the results of AP test and RP test for given coordinate. The figures show the desired points and the actual points for 3 times.
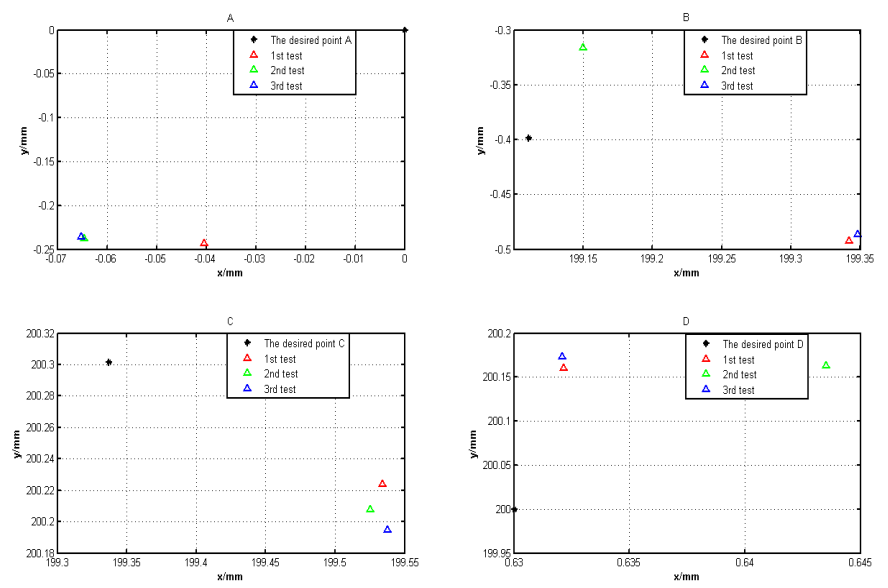

Fig. 4. Measuring results of welding robot in planar motion

\section{Conclusions}

To determine the position precision of the industry robot, the laser tracking measurement system is studied in the paper. The work overcomes the coupling of Spherical coordinate system, and establishes a Cartesian coordinate-based laser tracking measurement system. And we employ this system to measure coordinates of end-effector of welding robot. Meanwhile this paper presented the procedure of experimental evaluation. The performance indices were obtained using experimental tests. The result showed that, it is convenient and correct to employ the laser tracking measurement system to measure the positions of manipulators. The method can also provide the foundation for the robot pose process control.

\section{References}

[1] Wang Jia, Lu Gang. Development of Laser Automatic Tracing Space Coordinate Measurement System(1). Aviation Metrology \& Measurement Technology, 1997,17(3): 34-39

[2] Wang Jia, Lu Gang. Development of Laser Automatic Tracing Space Coordinate Measurement System(2). Aviation Metrology \& Measurement Technology, 1997,17(4): 34-38

[3] Yu Jia, Liu Yongdong. Main Principles and Technological Characteristics for Industrial Dynamic Tracking Measurement, Acta Metrologica Sinica, 2000, 21 (1): 34 39.

[4] Li Yongqian, Zhu Quanming. Status and Development of the Modern Precision Measurement Technology, Aeronautical Manufacturing Technology, 1999, (3) : 13 15

[5] FARO Provides, New Laser Scanner Photon and Software, Aeronautical Manufacturing Gechnology, 2009, (09)

[6] Li Xinghua, The Design of Laser Tracing System. 2003, Tianjing University: Tianjing

[7] AFNOR,Norme ISO9283: Robots manipulateurs industriels. AFNOR,1998 